arXiv:0706.1977 [astro-ph]

June 2007

\title{
Challenging the Cosmological Constant
}

\author{
Nemanja Kaloper ${ }^{1}$ \\ Department of Physics, University of California, Davis, CA 95616
}

\begin{abstract}
We outline a dynamical dark energy scenario whose signatures may be simultaneously tested by astronomical observations and laboratory experiments. The dark energy is a field with slightly sub-gravitational couplings to matter, a logarithmic self-interaction potential with a scale tuned to $\sim 10^{-3} \mathrm{eV}$, as is usual in quintessence models, and an effective mass $m_{\phi}$ influenced by the environmental energy density. Its forces may be suppressed just below the current bounds by the chameleon-like mimicry, whereby only outer layers of mass distributions, of thickness $1 / m_{\phi}$, give off appreciable long range forces. After inflation and reheating, the field is relativistic, and attains a Planckian expectation value before Hubble friction freezes it. This can make gravity in space slightly stronger than on Earth. During the matter era, interactions with nonrelativistic matter dig a minimum close to the Planck scale. However, due to its sub-gravitational matter couplings the field will linger away from this minimum until the matter energy density dips below $\sim 10^{-12} \mathrm{eV}^{4}$. Then it starts to roll to the minimum, driving a period of cosmic acceleration. Among the signatures of this scenario may be dark energy equation of state $w \neq-1$, stronger gravity in dilute mediums, that may influence BBN and appear as an excess of dark matter, and sub-millimeter corrections to Newton's law, close to the present laboratory limits.
\end{abstract}

\footnotetext{
${ }^{1}$ kaloper@physics.ucdavis.edu
} 
Understanding cosmic acceleration is the deepest problem of modern cosmology. It has profound implications both for fundamental physics and for the fate of the universe [1]. A range of ideas have been pursued to explain the acceleration, and to date in all of them, one is forced to fine tune some dimensional scales to accommodate cosmic acceleration now. This yields the 'Why Now' problem, which may be taken as a clue that we are missing something important in the formulation of the problem [2]. To compound the puzzle, to date we have noted other curious coincidences, such as the near matches between the scale of the cosmological constant, the dark matter density, the neutrino mass, and the laboratory limits on gravitational force, which are all controlled by a length scale of about a millimeter. While these may simply be numerical accidents, it is interesting to probe for deeper connections between them. We can pursue this by formulating models where cosmic acceleration has other direct observable consequences, as exemplified in [3]-[7].

The main problem in building such models is the range of mass scales which one needs for nontrivial dynamics. For example, to have a dynamical dark energy instead of the cosmological constant one needs ultralight degrees of freedom, say scalars, with masses $m_{\phi} \lesssim H_{0} \sim 10^{-33} \mathrm{eV}$. These must couple to matter significantly more weakly than gravity to avoid conflicts with Solar System tests [8]. On the other hand, laboratory tests constrain new fields to be heavier than about $10^{-3} \mathrm{eV}$, if they couple to matter gravitationally [9]. So to make dark energy detectable in laboratory searches and consistent with long range gravity, we need models where its mass changes by at least thirty orders of magnitude between the Earth and the extragalactic space. Indeed, if the masses of dark fields are fixed by the current laboratory bounds, we could integrate them out at scales below their masses and end up with dark energy practically indistinguishable from the pure cosmological constant, without a direct link to laboratory phenomena.

In this note we will outline a model of quintessence which may be within reach of future terrestrial searches for sub-millimeter corrections to Newton's law of gravity. It controls cosmology at largest scales with a very weak potential, logarithmic in the field value. Yet at shorter scales, due to large environmental masses as in $[10,11,12]$, this field could decouple at the scales probed by current laboratory tests, but perhaps just barely, so that it could be revealed by future probes. Its signatures, in addition to possible sub-millimeter gravitational effects, would include an equation of state $w \neq-1$, distinguishing it from the cosmological constant, stronger gravity in less dense mediums, which can influence BBN, and induce a weak spatio-temporal variation of Newton's constant, affecting structure formation and possibly simulating an excess of dark matter abundance over its actual density. This model could therefore be a useful benchmark for future observational explorations of the signatures of dark energy.

We start our discussion with a review of the mechanisms that make the masses of fields dependent on the medium in which they propagate [10]-[14]. They may provide a way around the usual decoupling argument, and are most simply formulated for models where the scalar couples to matter universally ${ }^{1}$, by interaction Lagrangians $\mathcal{L}_{\text {matter }}\left(g^{\mu \nu} e^{-2 \alpha \phi / M_{4}}, \Psi\right)$ like a Brans-Dicke field. In these cases, the effective potential controlling the propagation of

\footnotetext{
${ }^{1}$ Wider classes of models where the coupling changes from species to species were studied in [11].
} 
a field in a medium is given by

$$
V_{e f f}(\phi)=V(\phi)-T_{\mu}^{\mu} e^{\alpha_{w} \phi / M_{4}}
$$

where $V(\phi)$ is the potential in the vacuum and $T_{\mu}^{\mu} \propto-\rho$ is the trace of the stress energy of the environment ${ }^{2}$. The Planck mass $M_{4}$ and the dimensionless quantity $\alpha_{w}=(1-3 w) \alpha$ parameterize the couplings of the scalar to matter. Thus in stationary matter distributions, the minimum of the field $\phi$ is at $\phi_{*}$, where $\partial_{\phi} V_{\text {eff }}\left(\phi_{*}\right)=0$. The effective mass governing the dynamics of the field fluctuations about this environmental minimum $\phi_{*}$ is $m_{\phi}^{2}=\partial_{\phi}^{2} V_{e f f}\left(\phi_{*}\right)$. In distributions of matter with energy density $\rho$ and pressure $p, T^{\mu}{ }_{\mu}=-(\rho-3 p)$, setting how $\phi_{*}$ and $m_{\phi}^{2}$ will depend on the energy density of the environment. As the energy density changes, so will the location of the minimum $\phi_{*}$. Over cosmological time scales, the evolution of the zero mode is governed by

$$
\begin{aligned}
& 3 M_{4}^{2} H^{2}=\frac{\dot{\phi}^{2}}{2}+V+\rho e^{\alpha_{w} \phi / M_{4}}, \\
& \dot{\rho}+3(1+w) H \rho=0 \\
& \ddot{\phi}+3 H \dot{\phi}+\frac{\partial V_{e f f}}{\partial \phi}=0
\end{aligned}
$$

which come from the Einstein's equations and the $\phi$ field equation in homogeneous and isotropic, spatially flat FRW universes, that are a good approximation for our universe from just after the beginning of inflation onwards. The simplicity of the source terms is ensured by our conventions. Clearly, $\phi_{*}$ is not an exact solution to these equations, but will be a good approximation over time scales $t \ll 1 / H$, if $m_{\phi}>H$. From these equations, we can immediately find the condition when $\phi$ can yield cosmic acceleration. Acceleration is not automatic: even if $\rho$ is propping $\phi$ up on a slope of $V$, it changes due to cosmic expansion, and the field $\phi$ may slide down $V$ too fast to support cosmic acceleration over a Hubble time. Indeed, we can check immediately that for the example of nonrelativistic matter, if $\phi$ sits in the minimum of $V_{\text {eff }}$ the total energy density changes according to $\dot{H}=-\frac{\dot{\phi}^{2}}{2 M_{4}^{2}}-\frac{\rho}{2 M_{4}^{2}} e^{\alpha \phi / M_{4}} \simeq-\frac{3}{2} H^{2}$, which is clearly too fast to support acceleration.

The criteria for acceleration can be formulated by generalizing inflationary slow roll parameters to arbitrary fluids. Using critical energy density $\rho_{c r}=3 M_{4}^{2} H^{2}$ we see that the universe will accelerate if

$$
\epsilon=\left|\frac{\dot{\rho}_{c r}}{H \rho_{c r}}\right|<1
$$

Acceleration will last an e-fold or more if

$$
\eta=\left|\frac{\dot{\epsilon}}{3 H \epsilon}\right|<1
$$

sustaining potential dominance for at least a Hubble time. We can now find the conditions for acceleration as follows. Suppose first that $m_{\phi}>H$. Then Eq. (4) tells that $\phi$ will

\footnotetext{
${ }^{2}$ Our conventions are $M_{4}^{2} G_{\mu \nu}=T_{\mu \nu}$ for the Einstein's equations and $\delta S_{\text {matter }}=\frac{1}{2} \int d^{4} x \sqrt{\bar{g}_{4}} \bar{T}^{\mu \nu} \delta \bar{g}_{\mu \nu}$ for the stress energy tensor in the Brans-Dicke frame. We will define Einstein frame components of $\bar{T}_{\mu \nu}$ by $\rho, p=e^{3(1+w) \alpha \phi / M_{4}} \bar{\rho}, \bar{p}$, respectively, for reasons of simplicity, to be noted shortly.
} 
rapidly settle into the environmental minimum $\phi_{*}$, during a time scale $1 / m_{\phi}$ over which the Hubble friction is negligible. The direct evaluation of the $\epsilon$ parameter then shows that $\epsilon=\left[\dot{\phi}^{2}+(1+w) \rho e^{\alpha_{w} \phi / M_{4}}\right] / V$. Then approximating $\phi \sim \phi_{*}$, differentiating $\frac{\partial V_{\text {eff }}}{\partial \phi_{*}}$ with respect to time and squaring it yields $\dot{\phi}^{2} \simeq 9(1-3 w)^{2} \alpha_{w}^{2} \frac{H^{2} \rho e^{\alpha} w \phi / M_{4}}{M_{4}^{2} m_{\phi}^{4}} \rho e^{\alpha_{w} \phi / M_{4}}$. Further using $\epsilon \lesssim 1$ and $m_{\phi}>H$ yields $\dot{\phi}^{2}<9(1-3 w)^{2} \alpha_{w}^{2} \rho e^{\alpha_{w} \phi / M_{4}}$. Thus generically we can neglect $\sim \dot{\phi}^{2}$ terms in $\epsilon$, yielding $\epsilon \simeq(1+w) \rho e^{\alpha \phi / M_{4}} / V$. Using this to evaluate $\eta$ in the limit $m_{\phi}>H$, we find that $\eta \simeq(1+w)^{2}$. So when $m_{\phi}>H$, cosmic acceleration won't last longer than only a fraction of an e-fold unless the environment obeys $|w+1|<1$. But that means that an agent other than $\phi$ plays the role of dark energy, and $\phi$ is merely a spectator. Hence if $\phi$ is to be dark energy at any time, we must have

$$
m_{\phi} \lesssim H
$$

over the relevant scales. In particular, for our $\phi$ to explain cosmic acceleration now we need $m_{\phi} \lesssim H_{0}$ at horizon scales, unless we introduce some other dark energy by hand.

Now, the vacuum potential $V$ must satisfy some conditions in order to allow for a dynamical setup which won't violate experimental bounds on deviations from General Relativity, while still yielding something non-trivial. The allure of the chameleon mechanism is the environmental screening of the long range forces from matter interior to the mass distributions [12]. Namely, inside masses the environmental mass of the field $m_{\phi}$ is shifted up to a value much larger than in the vacuum, and so the chameleon forces of particles inside the distributions acquire efficient Yukawa suppressions, by the exponent of the depth of the source particle inside the mass distribution, in the units of $1 / m_{\phi}$. The suppressions die out for particles in the outer layer of the mass, of thickness roughly $\sim 1 / m_{\phi}(\phi)$ (which may have to be evaluated at some interpolating value of $\phi$ nearer to the boundary of the matter distribution, rather that its value $\phi_{*}$ in the core, to account for the variation of the homogeneous field mode through the matter distribution). This yields the net scalar force suppression relative to gravity by a factor of roughly $\sim m_{\phi}^{-1} / \mathcal{R}$, where $\mathcal{R}$ is the size of the source, even if the scalar is ultralight outside of the masses [12]. Clearly, the smaller the source, the less the suppression, and this is why for laboratory experiments, which work at a millimeter scale, this still translates to roughly $m_{\phi} \gtrsim 10^{-3} \mathrm{eV}$, for couplings of the order of $\mathcal{O}(1) \times M_{4}^{-1}$. Another important phenomenon concerns the effective gravitational coupling of matter. One sees immediately that environmental minima must obey $\left|\alpha \Delta \phi_{*}\right|<M_{4}$ over a wide range of scales, where $\Delta \phi_{*}$ is the shift of $\phi_{*}$ with the change of $\rho$. Otherwise, the effective gravitational coupling $G_{N \text { eff }} \sim \frac{1}{M_{4}^{2}} \exp \left(\alpha_{w} \phi_{*} / M_{4}\right)$ would change too much between the laboratory and, say, the atmosphere [12]. We should mention that the bounds from astrophysical gravitational fields may be weaker because of various model-dependent issues and systematics, such as the type, distribution and amount of dark matter et cetera.

This renders potentials dominated by $V \sim m^{2} \phi^{2}$ unsuitable for chameleonic dark energy model building, as follows. For a quadratic potential, the environmental minimum generated by couplings to nonrelativistic matter lies at $\frac{\alpha \phi_{*}}{M_{4}} e^{-\alpha \phi_{*} / M_{4}} \sim \frac{\alpha^{2} \rho}{M_{4}^{2} m^{2}}$, and the scalar mass is $m_{\phi}^{2}=m^{2}+\frac{\alpha^{2} \rho}{M_{4}^{2}} e^{\alpha \phi_{*} / M_{4}}$. If this field were quintessence, at cosmological scales where $\rho \sim M_{4}^{2} H_{0}^{2}$ its mass $m_{\phi}^{2}=m^{2}+\alpha^{2} H_{0}^{2} e^{\alpha \phi_{*} / M_{4}}$ must be smaller than $H_{0}^{2}$, as explained above, implying 
the same for the vacuum mass, $m^{2}<H_{0}^{2}$. But then, the environmental minimum would be at $\frac{\alpha \phi_{*}}{M_{4}} e^{-\alpha \phi_{*} / M_{4}}>\frac{\alpha^{2} \rho}{M_{4}^{2} H_{0}^{2}} \gg 1$ for relevant environments, implying that $\alpha \phi_{*} / M_{4}$ and therefore $G_{N \text { eff }}$ change too much with variations of $\rho$. Conversely, one could suppress variations of the effective Newton's constant either by taking $m^{2} \gg H_{0}^{2}$, or by taking $\alpha \rightarrow 0$, but then either the field $\phi$ should be integrated out at scales below $m$ and cannot be quintessence, or it would altogether decouple from matter and cease to behave as a chameleon.

Hence other potentials must be considered. Various specific examples were discussed in [12]-[18]. The works [12]-[15] employed vacuum potentials that can be approximated as powers $V \sim \frac{\lambda}{n} \phi^{n}$ for $n \neq 2$ (positive or negative!), yielding $V_{\text {eff }}(\phi)=\frac{\lambda}{n} \phi^{n}+\frac{1}{2} \rho e^{\alpha \phi / M_{4}}$. After adjusting the coupling $\lambda$ to satisfy $\alpha \phi_{*} \ll M_{4}$ at the minimum, that prevents large variations of $G_{N \text { eff }}$, the effective minimum is at $\phi_{*} \simeq\left(\frac{\alpha}{2 M_{4} \lambda}\right)^{1 /(n-1)} \rho^{1 /(n-1)}$. Around it, the scalar mass is dominated by $\partial_{\phi}^{2} V$ at the minimum, for $n \geq 2$, and is

$$
m_{\phi}^{2} \simeq(n-1) \lambda^{1 /(n-1)}\left(\frac{\alpha}{M_{4}}\right)^{\frac{n-2}{n-1}} \rho^{\frac{n-2}{n-1}}
$$

This formula breaks down for linear potentials with $n=1$, where the correct derivation yields $\gamma=1 / 2$. Indeed, for the linear potential $V=V_{0}-q \phi, \partial^{2} V=0$ and so the scalar mass is entirely an environmental effect: $m_{\phi}^{2} \simeq \frac{\alpha^{2} \rho}{M_{4}^{2}} e^{\alpha \phi / M_{4}} \sim \rho$. Thus generically

$$
m_{\phi} \propto \rho^{\gamma}
$$

where $\gamma=\frac{n-2}{2(n-1)}$, or $\gamma=1 / 2$ for $n=1$. When the matter couplings of $\phi$ are of the gravitational strength, $\alpha \sim 1$, this means that for all reasonable power law potentials, with integer powers, once the environmental mass $m_{\phi}$ is fixed by the laboratory bounds on Earth, $m_{\phi} \gtrsim 10^{-3} \mathrm{eV}$, for $\rho_{\text {Earth }} \sim \mathrm{g} / \mathrm{cm}^{3} \sim 10^{21} \mathrm{eV}^{4}$, it can decrease at most by a factor of

$$
\left(\frac{M_{4}^{2} H_{0}^{2}}{\rho_{\text {Earth }}}\right)^{\gamma} \simeq 10^{-33 \gamma}
$$

as the energy density changes to the cosmological background density. Having started at $m_{\phi} \gtrsim 10^{-3} \mathrm{eV}$, the effective environmental mass can therefore decrease down only to $m_{\phi} \gtrsim 10^{-3-33 \gamma} \mathrm{eV}$. For $\phi$ to be quintessence, suspended in slow roll on a potential slope at very large distance scales, this must be smaller than $H_{0} \sim 10^{-33} \mathrm{eV}$, which therefore requires $\gamma \geq 1$. Otherwise, the field $\phi$ will be too heavy to have any significant dynamics at the horizon scale, and dark energy must come from other quarters, if at all ${ }^{3}$, which is what happens with all integer powers.

The exception to this conclusion is the logarithmic potential $V \sim \ln \phi$. To see that it evades the arguments above, we note that to get the mass of $\phi$ for this case, we can take the limit of Eq. (8) when $n \rightarrow 0^{-}$. Then, $\gamma \rightarrow 1$, and so $m_{\phi} \sim \rho$. In this case, the effective mass will change by the full range of density ratio between the cosmological and terrestrial scales, spanning over thirty orders of magnitude. Hence, the logarithmic potential can give us a

\footnotetext{
${ }^{3}$ One can check that similar arguments also apply to, for example, exponential potentials. In that case, one also finds that the quintessence mass scales as (9) with $\gamma<1$ and that generically it is impossible to keep $\left|\alpha \phi_{*}\right|<M_{4}$ over a wide range of density variations.
} 
chameleonic, or changeling quintessence which could have evaded the laboratory searches for deviations from Newton's law at the current level of sensitivity, but may remain close to the bounds, within the reach of the future tests.

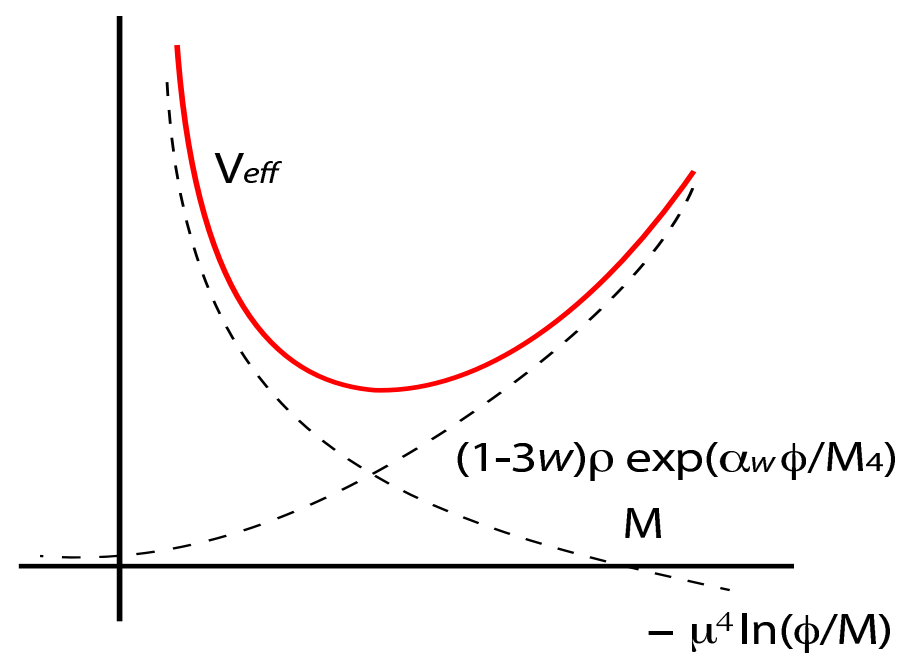

Figure 1: Environmental effective potential.

To explore the physics of our logarithmic dark energy changeling, we now turn to a specific model. Suppose that the vacuum potential of the scalar is $V=-\mu^{4} \ln (\phi / M)$, where ${ }^{4} M \gtrsim M_{4}$. Potentials like this may arise in theories with two conical extra dimensions, after their stabilization [20], or by integrating out some heavy fields which couple to the scalar $\phi$, such as in the MaVaNs models of [5] which also employ logarithmic potentials. However in contrast to $M a V a N s$, our $\phi$ couples universally to all 'light' matter, and dwells in a different regime, as exemplified by the sign and our choice of the scale $M$. As we will see later, we will need $\mu \sim 10^{-3} \mathrm{eV}$, which is usual for quintessence models that can fit the data. We won't commit to any particular mechanism explaining how such scales may arise (and in particular why there aren't larger corrections to $V$, which of course is the full cosmological constant problem that we can't solve yet [1]), instead focusing on their implications for observations. Nevertheless, we note that obtaining such potentials may only require tunings in the gravitational sector, if the scalar $\phi$ is a Brans-Dicke-like field, obeying weak equivalence principle, since there exists a Brans-Dicke frame to which matter couples universally. Then, the effective potential including the environmental correction from a medium obeying equation of state $p / \rho=w$ is

$$
V_{\text {eff }}(\phi)=-\mu^{4} \ln \left(\frac{\phi}{M}\right)+(1-3 w) \rho e^{\alpha_{w} \phi / M_{4}} .
$$

It is given in Figure (1) for a fixed value of $\rho$, and for $w<-1 / 3$. For $w=-1 / 3$ the environmental term is absent, whereas for $w>1 / 3$ it changes sign and convexity (since $\left.\alpha_{w}=(1-3 w) \alpha\right)$.

\footnotetext{
${ }^{4}$ We will work with $M$ not much greater than the Planck scale, to comply with the arguments about absence of ultraweak forces and trans-Planckian cutoffs in plausible UV completions of gravity [19].
} 
Let's examine cosmological history of such a theory, and see what are its predictions. We will work with the assumption that our universe was shaped by inflation, at some high scale $\Lambda \gg \mu^{4}$ and with $w \simeq-1$. Then, during inflation, the scalar field $\phi$ is controlled by $V_{\text {eff }}(\phi)=-\mu^{4} \ln \left(\frac{\phi}{M}\right)+4 \Lambda e^{4 \alpha \phi / M_{4}}$. The minimum of this potential is at $\frac{\alpha \phi_{*}}{M_{4}} \simeq \frac{\mu^{4}}{16 \Lambda} \ll 1$, and the effective scalar mass there is $m_{\phi}^{2} \simeq \frac{256 \alpha^{2} \Lambda^{2}}{M_{4}^{2} \mu^{4}} \gg H_{\text {inflation. }}^{2}$. In fact this is generically so large that the field $\phi$ is completely non-dynamical during inflation. It is frozen out extremely efficiently.

At the end of inflation, the energy in the inflaton potential $\Lambda$ will be converted into radiation. During this stage, the universe will rapidly become radiation-dominated, with $\rho_{\text {radiation }} \gg \rho_{\text {matter }}$. Ignoring the possibility of massive matter decays, we can place a bound on the ratio of $\rho_{\text {radiation }} / \rho_{\text {matter }}$ by scaling it up from matter-raditation equality to the reheating temperature: $\rho_{\text {radiation }} / \rho_{\text {matter }} \gtrsim T_{\text {reheating }} / \mathrm{eV}$, which can be as high as $10^{20}$ or so. In reality this ratio will be even higher because many of the nonrelativistic species today will have behaved as relativistic particles in the early universe. Now, the presence of massive particle species may generate a different effective potential for $\phi$, shifting the location of the environmental minimum. The environmental potential coming from nonrelativistic species is $V_{\text {eff }}(\phi)=-\mu^{4} \ln \left(\frac{\phi}{M}\right)+\rho_{\text {matter }} e^{\alpha \phi / M_{4}}$, with a minimum at $\frac{\alpha \phi_{*}}{M_{4}} \simeq \frac{\mu^{4}}{\rho_{\text {matter }}}$, and a mass around it $m_{\phi}^{2} \simeq \frac{\alpha^{2} \rho_{\text {matter }}^{2}}{M_{4}^{2} \mu^{4}} \simeq \frac{\alpha^{2} \rho_{\text {matter }}}{\mu^{4}} \frac{\rho_{\text {matter }}}{\rho_{\text {radiation }}} H_{\text {radiation }}^{2}$. During the radiation phase $\rho_{\text {radiation }} \gg \rho_{\text {matter }}$, and so $m_{\phi}^{2} \ll H_{\text {radiation. }}^{2}$ This minimum, if at all present, will be too shallow to affect cosmological dynamics of $\phi$.

Thus we can ignore $\rho_{\text {matter }}$ during the radiation epoch. The effective potential for $\phi$ changes to the pure logarithmic term, where the field is massless and initially close to the origin, where inflation left it: $\phi_{\text {inflation }} \simeq \frac{\mu^{4} M_{4}}{16 \Lambda \alpha}$. However generically the field will have a lot of kinetic energy after being released from its inflationary state. To see that, introduce $\tilde{\rho}=$ $\Lambda e^{4 \alpha \phi / M_{4}}$ as the total energy density during inflation. Just before the end of inflation, where $\Lambda$ starts to decay, the time variation of $\Lambda$ will pull along $\phi, \dot{\tilde{\rho}} \gtrsim \frac{4 \alpha \dot{\phi}}{M_{4}} \tilde{\rho}$, whence $\dot{\phi} \lesssim \frac{\dot{\tilde{\rho}}}{4 \alpha \tilde{\rho}} M_{4}$. With efficient reheating we can estimate $\underset{\tilde{\tilde{\rho}}}{\dot{\tilde{\rho}}} \simeq H_{\text {inflation }}$, so that $\dot{\phi} \lesssim \frac{M_{4} H_{\text {inflation }}}{4 \alpha}$, or $\dot{\phi}^{2} \lesssim \frac{\Lambda}{48 \alpha^{2}}$. While by no means precise, this argument at least shows that at the end of inflation, the field $\phi$ will generically convert a significant fraction of vacuum energy into its kinetic energy, by the universality of its couplings to all types of matter and equipartition of energy. The precise amount would depend on the model of inflation and reheating. Having so much kinetic energy after inflation is not dangerous for cosmology because it will dissipate quickly due to Hubble friction/redshift. Since we can neglect nonrelativistic matter at this stage, and because the potential energy density at this time is $V \sim \mu^{4} \ll \Lambda$, we can in fact ignore the effective potential altogether. As a result the field will evolve as a pure massless mode in a radiation-dominated universe, where it will stop more or less after a Hubble time, travelling a distance $\Delta \phi \sim \dot{\phi}_{\text {initial }} / H_{\text {inflation }} \lesssim \frac{M_{4}}{4 \alpha} \gg \phi_{\text {initial }}$ before it stops [21]. At that point, it will have an expectation value $\phi \lesssim \frac{M_{4}}{4 \alpha}$, a tiny potential energy, $V \lesssim \mu^{4} \ln \left(\frac{4 \alpha M}{M_{4}}\right)$, and a tiny mass ${ }^{5}$ $m_{\phi}^{2} \simeq \frac{\alpha^{2} \rho_{\text {matter }}}{M_{4}^{2}} \ll H_{\text {radiation }}^{2}$, giving a slightly stronger effective gravitational coupling $G_{N \text { eff }}$ to matter than to radiation, by at most a factor of about $\lesssim e^{1 / 4} \sim 1.28$ or so. For the rest of the radiation era, the field will simply just wait there.

\footnotetext{
${ }^{5}$ For as long as $\rho_{\text {matter }}>\mu^{4}$.
} 
We should comment here on the implications of the enhancement of $G_{N \text { eff }}$ for Big Bang nucleosynthesis. A difference between the value of Newton's constant in the early universe, and specifically at the time of BBN and its value measured presently in terrestrial experiments would affect relic abundances, and so BBN gives us strong limits on the variation of $G_{N}[22]$. However, our calculated maximal value of $G_{N \text { eff }}$ above, is the value of Newton's constant at nucleosynthesis as seen by nonrelativistic particles, with masses $m \gg \mathrm{MeV}$ at that time. Indeed, we recall that - as illustrated in e.g. Eq. (1) - the effective Newton's constant which a species sees is $G_{N \text { eff }} \sim \frac{1}{M_{4}^{2}} e^{\alpha_{w} \phi / M_{4}}$, where $\alpha_{w}=(1-3 w) \alpha$. Thus the relativistic particles, which are controlling the expansion rate of the universe at that time, would feel an effective Newton's constant much closer to its terrestrial value. Even the maximal value which we estimated above, felt by heavy particles, may be consistent with the new BBN bounds on $\Delta G_{N} / G_{N 0}$ that allow it to be $\sim 20 \%[23,24]$, although stronger bounds may be inferred from different data [25]. Hence BBN data may probe this aspect of our model, similarly to what happens in general scalar-tensor theories [10]. This should be explored in more detail. We need to also stress that the bounds from Oklo are easy to comply with. By the time the Oklo reactor started, the field would have settled into its terrestrial minimum, pulling $G_{N \text { eff }}$ down to its familiar value.

After radiation-matter transition, $\propto \rho_{\text {matter }}$ term in the effective potential will be of the order of $M_{4}^{2} H^{2}$. The environmental minimum for $\phi$ at the largest scales will become more prominent, and its location, as previously calculated, will be at $\frac{\alpha \phi_{*}}{M_{4}} \simeq \frac{\mu^{4}}{\rho_{\text {matter }}}$. Now, in dilute universe before structure formation, but after radiation-matter transition, $\rho_{\text {matter }}$ will be below $\mathrm{eV}^{4}$, approaching $\mu^{4}$ from above. This means, that the minimum has been shifting towards the Planckian values, where the field has been laying in wait. Yet, as long as $\alpha<1$, the field will not shift from where it went during the radiation epoch. The reason is that as long as $\rho_{\text {matter }}>\mu^{4}$, it's mass is still given by $m_{\phi}^{2} \simeq \frac{\alpha^{2} \rho_{\text {matter }}}{M_{4}^{2}}$. So by arranging $\alpha<1 / \sqrt{3}$, we can still keep $m_{\phi}^{2}<\frac{\rho_{\text {matter }}}{3 M_{4}^{2}}=H_{\text {matter }}^{2}$, holding the field up on the logarithmic slope by Hubble friction.

On the other hand, at shorter scales structure will begin to form around the primordial gravitational wells generated during inflation, where matter will agglomerate and the local matter density will increase manyfold over the uniform background value. In these regions, the environmental minima for $\phi$ will be closer to the origin and deeper, with $m_{\phi}^{2} \gg H_{m a t t e r}^{2}$. Hence where collapse began the field $\phi$ will fall back to the environmental minimum, oscillating around it instead of sticking to its post-inflationary value. In these regions, therefore, the field will behave as a component of cold dark matter, and its uniform energy density inside the region will begin to redshift as $\sim 1 / a^{3}$, yielding the scaling of $\phi \sim 1 / a^{3 / 2}$, similarly to unified dark matter models $[17,18]$. This stage of evolution can reduce the field value by as much as $\sim 10^{7}$ inside large scale overdensities. Moreover, at shorter scales gravitational cooling of the field [26] will lead to the collapse of the field energy to the core of the distribution, as in scalar field dark matter models, and to virialization with collapsing matter [27]. This will further reduce the value of the scalar field around the central overdensity to $\phi \ll M_{4}$, sweeping it into the center. Finally, where the matter overdensity reaches the scales of $\rho \sim 10^{6} \mathrm{eV}^{4}$ and beyond, the field mass will be $m_{\phi} \gtrsim 10^{-16} \mathrm{eV}$, so that the leftover field oscillations in time will occur at frequencies $\gtrsim \mathrm{sec}^{-1}$ about the minimum, so that we 
may replace it with its time average, $\phi_{*}$. So the long range effects of fields in these regions should be suppressed by the conspiracy between its environmental mass and the thin shell effect. Overall however one must be careful about picking the field boundary conditions in determining the long range forces as these depend sensitively on the evolution of matter and field distributions. To set up the long range fields, in general one therefore needs to look at the full history of the system. It is also possible that the field may leave some imprint in the large scale structure, since it will be more active in the beginning of the collapse. The precise description of these imprints is beyond the scope of this work, but we expect that because the imprints arise due to stronger gravity, they may affect our determination of dark matter abundance, leading us to overestimate the abundance of dark matter in structures which are at an early stage of their formation. Presumably this may lead to the possibility of direct astronomical tests and it would be interesting to develop further.

Back at cosmological scales, the evolution will eventually dilute $\rho_{\text {matter }}$ to below $\mu^{4}$. At this time, the universe will become dominated by the small residual potential energy in the field, $V \lesssim \mu^{4} \ln \left(\frac{4 \alpha M}{M_{4}}\right) \sim \mu^{4}$. The environmental minimum will shift to $\frac{\alpha \phi_{*}}{M_{4}}>1$. However, the effective field mass at the largest scales will change to $m_{\phi}^{2} \simeq \frac{\mu^{4}}{\phi^{2}}$, which is initially $m_{\phi}^{2} \simeq 16 \frac{\alpha^{2} \mu^{4}}{M_{4}^{2}}$. So the field will remain away from the minimum, and will start to slowly roll towards it as $\rho_{\text {matter }}$ dips below $\mu^{4}$. To ensure that the universe accelerates right away, we need to enforce Eq. (7). At this time, Eq. (7) translates to

$$
\alpha \lesssim \frac{1}{4 \sqrt{3}}
$$

Similarly, we must also demand that $V>0$, which implies $4 \alpha M>M_{4}$, and that the period of acceleration lasts at least an e-fold or so, $\Delta t \gtrsim 1 / H_{0}$. A stronger bound on $M$ comes about as follows. As time goes on and $\phi$ rolls down the logarithmic slope, the slow roll will improve, as $m_{\phi}^{2}$ is decreasing with the increase of $\phi$, as $m_{\phi}^{2} \simeq \frac{\mu^{4}}{\phi^{2}}$. Thus solving the field equations (2)-(4) in the slow roll regime, we find that

$$
\frac{\mu^{2} M_{4}}{\sqrt{3}} \Delta t \simeq \int_{\phi_{0}}^{\phi} d \phi \phi \ln ^{1 / 2}\left(\frac{M}{\phi}\right),
$$

where $\phi_{0}$ is the value of $\phi$ at the beginning of acceleration, $\phi_{0} \lesssim \frac{M_{4}}{4 \alpha}$. The integral is extremized by taking $\phi_{0}=\frac{M_{4}}{4 \alpha}$, and $\phi=M$, because the log potential will vanish there, and so if there are no higher order corrections that can prevent the potential from going negative, acceleration will only last until $\phi$ reaches $M$. Beyond that, acceleration will cease, and in fact the universe may even collapse, as has been recently studied in [28]. So substituting $\phi=M e^{-x / 2}$, the integral reduces to $\frac{M^{2}}{2^{3 / 2}} \int_{0}^{2 \ln \left(\frac{4 \alpha M}{M_{4}}\right)} d x \sqrt{x} e^{-x}$. By using $4 \alpha M>M_{4}$ and Eq. (12), we can maximize it with an Euler gamma function $\Gamma\left(\frac{3}{2}\right)$. The error is tolerable, as one can verify by using the saddle point approximation. Thus, the total duration of the late accelerating phase cannot be longer than $\Delta t \simeq \sqrt{\frac{3 \pi}{32}} \frac{M^{2}}{\mu^{2} M_{4}}$. The logarithmic plateau needs to be wide enough to accommodate at least an e-fold of inflation during this time, which, after setting $H_{0} \simeq \frac{\mu^{2}}{\sqrt{3} M_{4}}$ and requiring $H_{0} \Delta t \gtrsim 1$, implies that

$$
M \gtrsim\left(\frac{32}{\pi}\right)^{1 / 4} M_{4} \simeq 1.78 M_{4}
$$


This will suffice to explain the observed cosmic acceleration. We note that the criticisms of the MaVaNs model [29] (see also [30]) are easily circumvented here, since $\phi$ is in the slow roll regime, independently of the matter terms from the onset of acceleration.

Now if we don't take $M$ too large, avoiding UV cutoffs much higher than the Planck scale [19], the scalar may have matter couplings to within an order of magnitude of the gravitational couplings. To see it we can combine (12) and inequality $4 \alpha M>M_{4}$ into

$$
\frac{M_{4}}{4 M}<\alpha \lesssim \frac{1}{4 \sqrt{3}} .
$$

Since the scalar coupling to matter is governed by

$$
g_{\phi} \sim \frac{\alpha}{M_{4}},
$$

and its mass in terrestrial environments, where $\rho_{\text {matter }} \gg \mu^{4}$, is

$$
m_{\phi} \sim \frac{\alpha \rho_{\text {matter }}}{M_{4} \mu^{2}} \sim \frac{\alpha}{10} \mathrm{eV},
$$

when $M$ is not too large there remains a chance that $\phi$ could be within the reach of the future laboratory searches, after further improvements in sensitivity. Moreover, in this case $\phi$ will be rolling noticeably after an e-fold or so. Hence it would behave as $w \neq-1$ dark energy.

To summarize, we have delineated a dark energy model which, while tuned as it stands now, can be tested at several different observational fronts. It is based on a light scalar, with slightly sub-gravitational couplings to matter and a mass which depends on the environmental energy density. Outside of dense matter distributions this field will be light, and may yield significant long range effects. In particular, if it has logarithmic self-interaction potential, like those that can arise in theories with conical extra dimensions [20,31], or is generated radiatively [5], it can be quintessence, with mass $m_{\phi} \lesssim H_{0}$. At the largest scales however, this field will couple to matter in contrast to typical quintessence models, albeit slightly more weakly than gravity. In the early universe it will have an expectation value that is larger than in the terrestrial minima, which would make gravity slightly stronger. This can have consequences for BBN. During structure formation, before the field decouples in deeper potential wells around denser matter distributions, it may have affected cosmic structures. We have not analyzed this in detail here, and it would be very interesting to determine precisely what kind of signatures can arise. They may imitate an excess in the amount of dark matter. Finally, at the scales governing terrestrial physics, this field will become sufficiently massive so that its long range force may be suppressed by the thin shell effect discussed in the context of chameleons. Hence it may have avoided detection to date. However, its effects may be probed by future searches for sub-millimeter corrections to gravity. We believe that this represents an interesting framework for testing gravity and dark energy in a correlated manner. Testing models which involve correlations between modifications of gravity at short and long scales will probe the robustness of General Relativity and its greatest failure, the cosmological constant. It is therefore important to scrutinize such ideas further. Perhaps, ultimately, we might even end up getting surprised! 


\section{Acknowledgements}

We thank A. Albrecht, L. Knox, K. Olive, L. Sorbo, G. Steigman, S. Watson and especially J. Khoury and J. A. Tyson for interesting conversations. This work was supported in

part by the DOE Grant DE-FG03-91ER40674 and in part by a Research Innovation Award from the Research Corporation.

\section{References}

[1] S. Weinberg, Rev. Mod. Phys. 61, 1 (1989).

[2] N. A. Bahcall, J. P. Ostriker, S. Perlmutter and P. J. Steinhardt, Science 284, 1481 (1999); I. Zlatev, L. M. Wang and P. J. Steinhardt, Phys. Rev. Lett. 82, 896 (1999); N. Arkani-Hamed, L. J. Hall, C. F. Kolda and H. Murayama, Phys. Rev. Lett. 85, 4434 (2000).

[3] S. R. Beane, Gen. Rel. Grav. 29, 945 (1997).

[4] T. Damour, F. Piazza and G. Veneziano, Phys. Rev. D 66, 046007 (2002); Phys. Rev. Lett. 89, 081601 (2002).

[5] R. Fardon, A. E. Nelson and N. Weiner, JCAP 0410, 005 (2004); JHEP 0603, 042 (2006); D. B. Kaplan, A. E. Nelson and N. Weiner, Phys. Rev. Lett. 93, 091801 (2004).

[6] P. Gu, X. Wang and X. Zhang, Phys. Rev. D 68, 087301 (2003); X. J. Bi, B. Feng, H. Li and X. m. Zhang, Phys. Rev. D 72, 123523 (2005).

[7] L. J. Hall, Y. Nomura and S. J. Oliver, Phys. Rev. Lett. 95, 141302 (2005); R. Barbieri, L. J. Hall, S. J. Oliver and A. Strumia, Phys. Lett. B 625, 189 (2005).

[8] S. M. Carroll, Phys. Rev. Lett. 81, 3067 (1998); C. F. Kolda and D. H. Lyth, Phys. Lett. B 458, 197 (1999); T. Chiba, Phys. Rev. D 60, 083508 (1999).

[9] E. G. Adelberger, B. R. Heckel, S. Hoedl, C. D. Hoyle, D. J. Kapner and A. Upadhye, Phys. Rev. Lett. 98, 131104 (2007); D. J. Kapner, T. S. Cook, E. G. Adelberger, J. H. Gundlach, B. R. Heckel, C. D. Hoyle and H. E. Swanson, Phys. Rev. Lett. 98, 021101 (2007); see also E. G. Adelberger, B. R. Heckel and A. E. Nelson, Ann. Rev. Nucl. Part. Sci. 53, 77 (2003).

[10] T. Damour and K. Nordtvedt, Phys. Rev. Lett. 70, 2217 (1993); Phys. Rev. D 48, 3436 (1993).

[11] T. Damour and A. M. Polyakov, Nucl. Phys. B 423, 532 (1994); Gen. Rel. Grav. 26, 1171 (1994).

[12] J. Khoury and A. Weltman, Phys. Rev. Lett. 93, 171104 (2004); Phys. Rev. D 69, 044026 (2004). 
[13] G. R. Dvali, G. Gabadadze and M. A. Shifman, Mod. Phys. Lett. A 16, 513 (2001).

[14] S. S. Gubser and J. Khoury, Phys. Rev. D 70, 104001 (2004).

[15] P. Brax, C. van de Bruck, A. C. Davis, J. Khoury and A. Weltman, Phys. Rev. D 70, 123518 (2004).

[16] H. Wei and R. G. Cai, Phys. Rev. D 71, 043504 (2005); T. Biswas, R. Brandenberger, A. Mazumdar and T. Multamaki, Phys. Rev. D 74, 063501 (2006).

[17] A. Y. Kamenshchik, U. Moschella and V. Pasquier, Phys. Lett. B 511, 265 (2001).

[18] A. Fuzfa and J. M. Alimi, Phys. Rev. Lett. 97, 061301 (2006); Phys. Rev. D 73, 023520 (2006); arXiv:astro-ph/0702478.

[19] M. Dine, arXiv:hep-th/0107259; T. Banks, M. Dine, P. J. Fox and E. Gorbatov, JCAP 0306, 001 (2003); N. Arkani-Hamed, L. Motl, A. Nicolis and C. Vafa, arXiv:hepth/0601001.

[20] N. Arkani-Hamed, L. J. Hall, D. R. Smith and N. Weiner, Phys. Rev. D 62, 105002 (2000); M. J. May and R. Sundrum, Phys. Rev. D 69, 104010 (2004).

[21] N. Kaloper and K. A. Olive, Astropart. Phys. 1, 185 (1993); A. A. Tseytlin and C. Vafa, Nucl. Phys. B 372, 443 (1992); T. Barreiro, B. de Carlos and E. J. Copeland, Phys. Rev. D 58, 083513 (1998); L. Kofman, A. Linde, X. Liu, A. Maloney, L. McAllister and E. Silverstein, JHEP 0405, 030 (2004); S. Watson, Phys. Rev. D 70, 066005 (2004); R. Brustein, S. P. de Alwis and P. Martens, Phys. Rev. D 70, 126012 (2004); N. Kaloper, J. Rahmfeld and L. Sorbo, Phys. Lett. B 606, 234 (2005); T. Barreiro, B. de Carlos, E. Copeland and N. J. Nunes, Phys. Rev. D 72, 106004 (2005); S. Cremonini and S. Watson, Phys. Rev. D 73, 086007 (2006); B. Greene, S. Judes, J. Levin, S. Watson and A. Weltman, arXiv:hep-th/0702220.

[22] G. Steigman, Nature 261, 479 (1976); A. M. Boesgaard and G. Steigman, Ann. Rev. Astron. Astrophys. 23, 319 (1985).

[23] C. J. Copi, A. N. Davis and L. M. Krauss, Phys. Rev. Lett. 92, 171301 (2004).

[24] R. H. Cyburt, B. D. Fields, K. A. Olive and E. Skillman, Astropart. Phys. 23, 313 (2005).

[25] G. Steigman, Int. J. Mod. Phys. E 15, 1 (2006).

[26] E. Seidel and W. M. Suen, Phys. Rev. Lett. 66, 1659 (1991); Phys. Rev. Lett. 72, 2516 (1994).

[27] T. Matos and L. A. Urena-Lopez, Class. Quant. Grav. 17, L75 (2000); T. Matos and L. A. Urena-Lopez, Phys. Rev. D 63, 063506 (2001); M. Alcubierre, F. S. Guzman, T. Matos, D. Nunez, L. A. Urena-Lopez and P. Wiederhold, Class. Quant. Grav. 19, 5017 (2002); F. S. Guzman and L. A. Urena-Lopez, Phys. Rev. D 69, 124033 (2004). 
[28] R. Kallosh, J. Kratochvil, A. Linde, E. V. Linder and M. Shmakova, JCAP 0310, 015 (2003).

[29] N. Afshordi, M. Zaldarriaga and K. Kohri, Phys. Rev. D 72, 065024 (2005).

[30] O. E. Bjaelde, A. W. Brookfield, C. van de Bruck, S. Hannestad, D. F. Mota, L. Schrempp and D. Tocchini-Valentini, arXiv:0705.2018 [astro-ph].

[31] N. Kaloper, arXiv:hep-th/0702206. 